\title{
A cross-sectional study for assessment of audio-visual reaction time in KSRTC drivers of age group 20-40 years in Belgaum city with respect of working hours
}

\author{
Harpreet K*, Raichur R. N.**
}

* Lecturer of Physiology, USM-KLE-IMP, KLE University, Belgaum, **Prof Physiology, J.N.Medical College, Belgaum

Indian J Sleep Med $2011 ; 6.4,135-139$

\begin{abstract}
Background: The quickness in any work in our life corresponds to the speed with which body respond to external stimuli. And quickness is very essential in profession like driving, in which faster level of response is one of measure by which number of road traffic accidents can be reduced. Audio-visual reaction is an important test for drivers.

Objectives: To compare Audio-Visual reaction time in day time \& night time working drivers.

Methods: Fifty age \& sex matched, drivers of age 20-40 years working in KSRTC, Belgaum in day and night hours that fits in inclusion criteria are taken after informed consent. A through clinical examination was done \& Audio -Visual Reaction time- is measured by Audio-Visual Analyzer.
\end{abstract}

Results: Auditory reaction time is shorter then visual reaction time.

Conclusion: And this test is an important test for assessment of quickness \& coordination of visual and auditory responses, which is utmost important in drivers to reduces the road traffic accidents.

Keywords: Visual reaction time, Auditory reaction time.

\section{Introduction}

$\mathrm{E}$ very action we make is an adjustment or reaction to some condition or stimulus either in theworld about us or in our own body. Our emotions, attention, memories and all other reactions we make are responses to stimuli which play upon $\mathrm{us}^{1}$. It is well known to all of us that our responses are prompt when we are attentive. Present day routine modern living involves a number of activities that have to performed

\section{Address for correspondence}

\section{Dr Harpreet Kour}

Lecturer of Physiology, USM-KLE-IMP, KLE

University, Belgaum-590010

Mobile no.: 9620850326

Off: 0831-2455557, Fax no: 0831-2455558

E-mail id: harpreet.kour@yahoo.co.in precisely and promptly. In everyday life, men have to respond often to numerous diverse situations often instaneously. For, instance for an automobile driver applying brakes in a fraction of second or for a factory worker on a machine, quick maneuvering is must and may be lifesaving ${ }^{2}$. In case of drivers, quick response is one of the very important measure by which the number of accidents can be prevented. A number of studies have identified sleep disturbances, ischemic heart diseases, peptic ulcers, reproductive hazards and hypertension to be the main health problems of shift workers ${ }^{3}$.

Night shift workers are required to stay awake when their circadian rhythms are preparing them for sleep, and to sleep when preparing for wakefulness. People who work at night often complain of symptoms similar to those of "jet lag syndrome" and the cause is believed to be similar. With continued night work, some workers 
find the inconvenience and malaise unacceptable ${ }^{4}$.

Psychologic disturbances during night shift work were associated with altered cardiovascular and endocrine responses in healthy nurses ${ }^{5}$. Older night workers are more adversely affected than younger night workers ${ }^{6}$. The earlier studies done on reaction time indicate auditory reaction time is faster than visual reaction time ${ }^{7}$. In one study it has been reported that red colour elicits a faster response compared to green ${ }^{8}$.One study shows that sleep deprivation increases reaction time ${ }^{9}$. There are a few studies looking at the effect of shift work on reaction time in certain occupations. Reaction time means time taken by an individual to react to external stimulus. It provides an indirect index of processing capability of the central nervous system and also a simple means of determining sensorimotor performances ${ }^{10}$. Reaction time has physiological significance and is a simple and noninvasive test of function for the peripheral as well as central structures ${ }^{11}$.

Many studies has been conducted on audio-visual reaction time in healthy subjects, in different age groups, in athletes and other sports, smokers, tobacco chewers, etc. But there are only a few studies on audio-visual reaction time in case of drivers. The present study was undertaken; keeping in view that the auditory- visual reaction is an utmost important test for the drivers, as it has a role in a prevention of road traffic accidents, assessing quickness $\&$ coordination of visual and auditory responses. This study was an attempt to find out the influence of night duty as compared to day duty reaction time in drivers.

\section{Objectives}

1. To find out difference between auditory \& visual reaction time in drivers

2. To find audio-visual reaction time in drivers with respect of day time $\&$ night time shifts.

\section{Materials \& Methods}

The study protocol was prepared and approval was taken from the Institutional Ethical Committee.

Fifty age \& sex matched, drivers of age 20-50 years working in KSRTC, Belgaum in day hours(short route drivers) and 50 age $\&$ sex controlled drivers of age 2050 years working in KSRTC, Belgaum in night hours (long route drivers) who fit the inclusion criteria. A through clinical examination was done. A selfadministered questionnaire to record personal data was given.

\section{Inclusion criteria}

1. All subjects are taken from KSRTC, Belgaum.

2. All the subjects included are healthy, nonalcoholic, nonsmokers or non-tobacco chewer.

3. All the subjects had no auditory or visual disturbances.

\section{Exclusion criteria:}

1. Subjects who are alcoholic, smokers or with auditory or visual disturbances.

2. Subjects suffering from any chronic disease.

The subjects were briefed about the study protocol and informed consent was obtained from them.

The apparatus used in this study was the portable research reaction timer with two response choices measuring VRT and ART.

Specifications of reaction timer:

1. Inbuilt chronoscope -4 digit chronoscope with least count of $1 / 1000$ seconds.

2. It works on -230 volts AC.

Each subject was made familiar with the apparatus and the procedure to alleviate any fear or apprehension. ART and VRT were measured in a quiet room. The tests were done with the subject sitting comfortably in a chair. ART and VRT were measured by reaction time instrument. Accuracy of this instrument is \pm one digit. All the subjects were right handers and used their right hand to press the switch to stop the quartz clock of the apparatus. From the auto-display reaction time was noted. ${ }^{9}$

1. Auditory reaction time- by audio-visual analyzer

2. Visual reaction time- by audio-visual analyzer

Subjects were presented randomly with 2 visual stimuli i.e. red $\&$ green light and 2 auditory stimuli i.e. high pitch and low pitch sound stimuli. The significance of difference of VRT and ART during day duty and night duty was studied with the use of standard error of difference between two means. Three readings of each stimulus were noted after giving three practical trials 
and the lowest reading was taken as the reaction time ${ }^{12-}$ ${ }^{14}$. To test whether there was any significant difference during day and night duty with reference to auditory reaction time and visual reaction time, standard error of difference between two means were applied. The statistical difference was determined by ' $Z$ ' test.

Table1: Comparison of ART and VRT in short and long route drivers.

\begin{tabular}{|l|l|l|ll|}
\hline Parameters & $\begin{array}{l}\text { Short route } \\
\text { drivers } \\
(\text { MEAN } \pm \text { SD })\end{array}$ & $\begin{array}{l}\text { Long route } \\
\text { drivers } \\
(\text { MEAN } \pm \text { SD })\end{array}$ & $\begin{array}{l}\text { 'z } \\
\text { value' }\end{array}$ & $\begin{array}{l}\text { 'P } \\
\text { value' }\end{array}$ \\
\hline ART Right & $202.4 \pm 27.77$ & $203.68 \pm 23.11$ & 0.35 & $>0.05$ \\
\hline ART Left & $204.62 \pm 28.23$ & $204.61 \pm 22.07$ & 0.21 & $>0.05$ \\
\hline $\begin{array}{l}\text { VRT Red } \\
\text { colour Right }\end{array}$ & $211.46 \pm 32.26$ & $214.35 \pm 26.63$ & 0.69 & $>0.05$ \\
\hline $\begin{array}{l}\text { VRT Red } \\
\text { colour Left }\end{array}$ & $213.99 \pm 32.66$ & $216.42 \pm 27.27$ & 0.58 & $>0.05$ \\
\hline $\begin{array}{l}\text { VRT Green } \\
\text { Colour Left }\end{array}$ & $214.79 \pm 29.47$ & $220.37 \pm 28.77$ & 1.35 & $>0.05$ \\
\hline $\begin{array}{l}\text { VRT Green } \\
\text { Colour Left }\end{array}$ & $220.86 \pm 37.93$ & $221.65 \pm 30.88$ & 0.16 & $>0.05$ \\
\hline
\end{tabular}

\section{Results}

Results were analyzed by ' $z$ ' test $\&$ student's t' test.

\section{Graph 1:}

There was no statistically significant difference between VRT to red light, green light and both combined during day duty and night duty. There was no statistically significant difference between ART for high pitch, low pitch. VRT during day duty were more than ART during day duty, difference between two were found statistically significant. VRT during night duty were more than ART during night duty, but difference between the two were not statistically significant.

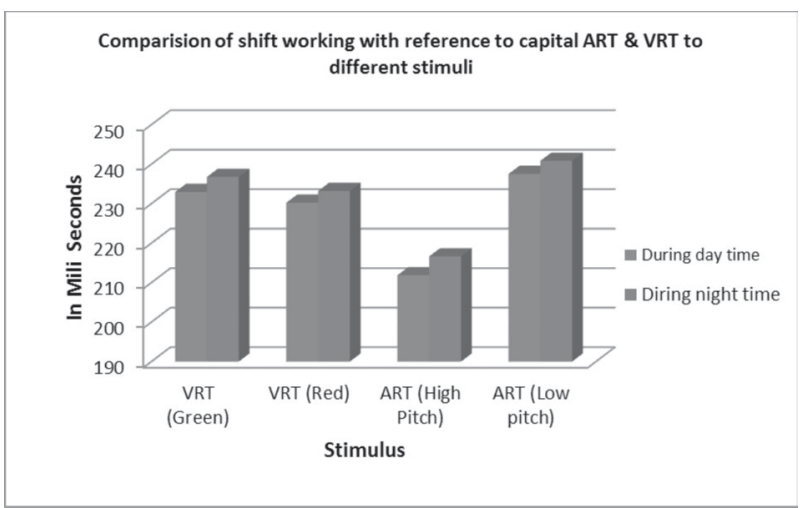

Values are mean $\pm S E .{ }^{*} P<0.05 ;{ }^{*} P>0.05$.
Graph II:

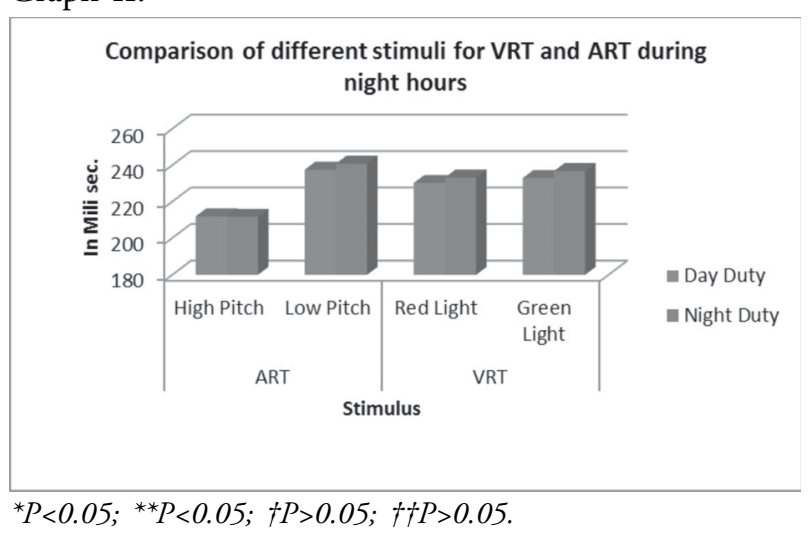

\section{Discussion}

In the present study auditory reaction time is shorter than visual reaction time. The reason being that for auditory reaction time, sound entering the ear can reach appropriate receptors with no particular loss of time for visual reaction time.

For visual reaction time the rods and cones are not excited by light directly and the intervening photochemical process takes appreciable time. It is shown that when sound is applied to the ear of cat, activity begins in the auditory nerve with latency of 1-2 ms whereas when a light is thrown into the eye, activity reaches visual cortex in $20-40 \mathrm{~ms}$, but, when the retina is by passed by direct electrical stimulation for the optic nerve, the cortical latency is as little as $2-5 \mathrm{~ms}$. Compared with the ear the eye takes long time to get its message started along the nerve to brain. Thus, one can account for most and perhaps all of the differences between visual and auditory reaction time ${ }^{2}$. Our observations are in agreement with studies by Boring et al $(1955)^{7}$, Madanmohan et al(1992) ${ }^{15}$, Malathi and Parulkar $(1989)^{16}$, Kapoor et al (1993) ${ }^{17}$, Saha et al (1996) ${ }^{18}$ and Singh et al (1994) ${ }^{19}$. Only Dhangauri et al $(1994)^{8}$ obtained higher ART values than VRT values in their study.

There is no significant difference found in VRT during day duty and night duty and ART during day duty and night duty in this present study (Graph I). It indicates that shift working has no effect on reaction time (auditory and visual), which means it has no effect on sensorimotor performance or cognitive functions. This can be explained on the basis of mechanism of adaptation to shift work and it may be associated with fewer problems encountered while working shifts. There are various 
factors that modulate adaptation to shift work. Workers who choose shift work for reasons such as schooling, childcare, or pay differential are likely to adapt more easily. However individuals vary in their ability to adjust to shift work. Many individuals suffer few or transient problems. Some are unable to adjust at all; a phenomenon termed as shift work intolerance ${ }^{20}$. This finding implies the potential of using perceived adaptation as a possible proxy for the health, wellbeing, and on-the-job alertness of shift workers ${ }^{21}$.

The study of Binks et al, found that 34-36 hours of total sleep deprivation did not affect performance on any of the cognitive tests ${ }^{22}$. The study performed by Bartle et al (1988) and Pilcher et $\mathrm{al}^{20 \& 23}$ confirms that acute sleep deprivation of less than 4 hours alters mood state but does not change performance in test situations in which concentration, clear thinking, and problem solving are important. Lack of 4 hours of uninterrupted sleep within the previous 24 hours does result in increased subjective fatigue and is associated with decreased motivation. However this effect does not necessarily lead to a decrease in performance ${ }^{20 \& 23}$.

In contrast, McCarthy et al ${ }^{24}$ in his study revealed significant effects on both cognitive and physiological measures including reaction time of sleep deprivation. Their findings reveals that sleep deprivation decreased subject's attention \& responsivity to new information and simultaneously reduced the efficiency of their cognitive processing. The studies done by Takeyama $\&$ Hirose $^{25-26}$ showed that modified night shift with restful power naps alleviated subjective fatigue, and improved physiological function which is often adversely affected by night workload ${ }^{25-26}$.

In our study, difference between VRT during day duty and ART during day duty were found statistically significant. VRT during night duty and ART during night duty were not statistically significant although VRT during night duty were greater than ART during night duty (Graph I). Reaction times are increasingly longer for responses evoked by proprioceptive, auditory, and visual stimuli respectively. The cause of visual reaction time being greater than auditory reaction time is not very clear though almost all of the research done in reaction time has reached the same conclusion. Most likely it is due to the fact that the visual reaction time involves chemical changes as mentioned earlier. Also the visual pathway involves many collateral pathways to various association areas and hence a greater delay in comprehension of visual stimulus as it is interpreted in a more complex and elaborate fashion.

There was significant difference in the auditory reaction time to high and low pitch sound stimuli during both day and night duty in hospital employees. ART to high pitch were lower than ART to low pitch (Graph II). It could be due to high intensity of high pitch sound which attracts early response. Shenvi et al (1994) ${ }^{27}$ showed no significant difference in the auditory reaction time to high and low pitch sound stimuli, although ART to high pitch were lower than ART to low pitch sound among the boys ${ }^{27}$.VRT to red light were lower than VRT to green light during both day and night duty, but it was not significant (Graph II). In study of Shenvi et al (1994) explained on the basis of Trichromatic theory of colour vision that there was significant difference in the visual reaction time to red and green light stimuli.

\section{Conclusion}

There was no significant difference in VRT during day duty and night duty and ART during day duty and night duty. The study shows that shift work has no effect on reaction time, in other words it has no effect on sensorimotor performance. Our findings could be explained on the basis of observations that adaptation to reduced sleep is possible and the factors connected with perceived adaptation might also be used to devise intervention strategies for improving shift work situations.

\section{References}

1. Joseph Tiffin, Federic B Knight and Eston Jackson Asher, The Psychology of normal people, $2^{\text {nd }}$ edition, D.C Health and company, Boston; 1946; 415-431.

2. Woodworth and Schlosberg, Experimental Psychology, $5^{\text {th }}$ edition, Oxford and IBH publication Co PVT Ltd; New Delhi: 1971: 8-42.

3. Cobb S, Rose RM (1973) Hypertension, peptic ulcer and diabetes in traffic controllers. JAMA, 224, 489-92.

4. Waterhouse JM. Altered time. In: Case RM, ed. Variations in Human Physiology. Manchester: Manchester University Press; 1985: 134-157.

5. Munakata $\mathbf{M}$, Ichii S, Nunokawa T et al. Influence of night shift work on psychological state and cardiovascular and neuroendocrine responses in healthy nurses. Hypertens Res 2001; 24: 25-31.

6. Pires MLN, Teixeira CW, Esteves AM, et al. Sleep, ageing and night work. Braz J Med Biol Res 2009; 42: 839-843. 
7. Dixit Yb, Assessment of Mental Alertness after Administration of various Phenothiazines, J Indian Med Assoc1964 Feb 16;42:173-5.

8. Dhangauri Shenvi and Padma Balasubramaian, A Comparitive Study of Visual and Auditory reaction times in males and females, Journal of Physiology and pharmacology, 1994:volume 38(3):229-231.

9. Corsi-cobrera M, Arce C, Ramos J, Lorenzo I and Guevara M.A. Time course of reaction time and EEG while performing a vigilance task during total sleep deprivation. Sleep 1996 ; 19: 563-569.

10. Madan Mohan, D.P.Thombare, Ashok Kumar Das N.Subramanian and S. Chandrasekar, Reaction time in Clinical Diabetes, Indian Journal of Physiology and Pharmacology, 1984:Volume28(4):311-314.

11. Das S, Gandhi A, Mondal S. Effect of premenstrual stress on audiovisual reaction time and audiogram. Ind $J$ Physiology Pharmacology 1997; 41: 67-70.

12. Namrata, Asha G, Sunita M and Shashi Narayan, Effect of iron deficiency anemia on Audiovisual reaction time in adolescent girls. Indian J Physiology Pharmacology 2011 ; 55 (1) : 53-59

13. Namita, Din Prakash R and Dhangauri N. Shenvi, Effect of Shift Working on Reaction time in Hospital Employees, Indian J Physiology Pharmacology 2010; 54 (3) : 289-293

14. Chinmay Shah, P A Gokhale, H.B. Mehta, Effect of Mobile Use on Reaction Time, Al Ameen J Med Sci (2010) 3(2): 160 164.

15. Madan Mohan, D.P. Thombare, BharathiBalaKumar, T.K.Nambinarayan, Sachin T, N.Krishnamoorthy and A.Chandrbose, Effect of yoga training on reaction time, respiratory endurance and muscle strength, Indian J. of Physiology and Pharmacology 1992:36(4):229-223.

16. A.Malati and Vidya G.Parulkar, Effect of Yogasana on the visual and auditory reaction time, Indian J. of Physiology and Pharmacology 1989:33(1): 100-112.

17. Raj Kapoor, S.H.singh and A.gandhi, Autonomic functions and audiovisual reaction time in heroin addicts, Indian J. of Physiology and Pharmacology 1993:37(3):209-212.
18. S.Saha, A.Gandhi, S.Das, P Kaur and S.H.Singh, Effect of noise stress on some cardiovascular parameters and audiovisual reaction time, Indian J. of Physiology and Pharmacology 1996:40(1):35-40

19. M.M.Singh, Gomti Devi and N.S. Devi. Study of Visual and Auditory Reaction time in Karate Trainee, Indian J. of Physiology and Pharmacology 1984:28(5): Supplement - 133.

20. Bartle EJ, Sun JH, Thompson L, Light Al, McCool C, Heatson $\mathrm{S}$. The effects of acute sleep deprivation during residency training. Surgery 1988; 104: $311-316$.

21. Masaya.T, Takeshi.T Naoko.T, Keiko.M, Yoshiko.K, Lawrence.S and Hiroyasu.I, Modifying Effects of Perceived Adaptation to Shift Work on Health, Wellbeing, and Alertness on the Job among Nuclear Power Plant Operators Industrial Health 2005, 43, 171-178

22. Binks PG, Waters WF, Hurry M. Short-term sleep deprivation does not selectively impair higher cortical functioning. Sleep 1999; 22: 328-334.

23. Pilcher JJ, Huffcutt Al. Effects of sleep deprivation on performance: A meta-analysis. Sleep 1996; 19: 318-326.

24. McCarthy ME, Waters WF. Decreased attentional responsivity during sleep deprivation: Orienting response latency, amplitude, and habituation. Sleep 1997; 20: 115123

25. Takeyama H, Itani T, Tachi N, Sakamura O, Murata K, Inoue $\mathrm{T}$, et al. Effects of a modified ambulance night shift system on fatigue and physiological function among ambulance paramedics. J Occup Health 2009; 51: 204209.

26. Hirose T. An occupational health physician's report on the improvement in the sleeping conditions of night shift workers. Industrial Health 2005; 43: 58-62.

27. Shenvi D, Balasubramanian P. A comparative study of visual and auditory reaction time in males and females. Ind $J$ Physiol Pharmacol 1994; 38: 229-231. 